Mots. Les langages du politique

Présidentielle 2007. Scènes de genre

\title{
Les femmes politiques à la télévision. Quel accès à la parole légitime?
}

Sheila Perry

\section{(2) OpenEdition \\ Journals}

Édition électronique

URL : https://journals.openedition.org/mots/19085

DOI : $10.4000 /$ mots. 19085

ISSN : 1960-6001

Éditeur

ENS Éditions

Édition imprimée

Date de publication : 1 juillet 2009

Pagination : 103-118

ISBN : 978-2-84788-151-6

ISSN : 0243-6450

Référence électronique

Sheila Perry, "Les femmes politiques à la télévision. Quel accès à la parole légitime? 》, Mots. Les langages du politique [En ligne], 90 | 2009, mis en ligne le 01 juillet 2011, consulté le 22 avril 2022. URL : http://journals.openedition.org/mots/19085; DOI : https://doi.org/10.4000/mots.19085 


\section{Sheila Perry}

\section{Les femmes politiques à la télévision. Quel accès à la parole légitime?}

Depuis les années quatre-vingt-dix, la sous-représentation politique des femmes en France est communément admise et quasi universellement déplorée. Des divergences d'opinion apparaissent quant aux mesures à adopter pour résoudre ce problème, mais le constat d'une disparité entre le nombre d'hommes et de femmes élus est indéniable, et ceci malgré l'adoption des lois sur la parité. Au lendemain des élections législatives de 2007 , il y avait 18,5\% de députées, $16,9 \%$ de sénatrices, $30 \%$ de conseillères municipales, 47,6 \% de conseillères régionales, $10,8 \%$ de femmes maires et une seule présidente de conseil régional sur 22, sans parler des instances politiques - conseils généraux, conseils de communautés urbaines ou d'agglomération, gouvernement (33\% de femmes en 2007) - que ces lois ne régissent pas (ou ne régissaient pas encore) avant 2008. La persistance de la domination masculine dans le champ politique est telle que, en 2005 (quand toutes les assemblées concernées par la parité politique s'étaient déjà renouvelées au moins une fois), Grégory Derville et Sophie Pionchon ont pu parler de l'«invisibilité» des femmes politiques, qu'ils attribuent à «la prégnance de représentations sociales qui sont largement antagonistes avec l'engagement politique au féminin » (Derville, Pionchon, 2005, p. 53). Représentations sociales qui sont sexuellement différenciées et largement relayées par la presse écrite (Holmes, 1988; Sourd, 2005).

Dans cet article, nous proposons d'examiner la part de responsabilité de la télévision dans l'invisibilité des femmes politiques ${ }^{1}$. Cet objectif peut paraitre paradoxal tant la télévision a pour mission de montrer, de rendre visible le monde réel, qu'il soit politique, économique, social ou culturel. Média national par excellence, la télévision permet aux citoyen(ne)s de toute la France de connaitre et de reconnaitre le visage de ceux et de celles dont le mandat s'exerce sur un territoire géographique délimité. Les hommes et les femmes politiques les plus «en vue» le sont surtout grâce à la télévision.

Université de Nottingham, sheila.perry@nottingham.ac.uk.

1. Cet article est issu de recherches dont les premiers résultats ont été publiés dans deux articles (Perry, 1995, 2000). 
Comment, donc, ce média peut-il être responsable de l'invisibilité des femmes politiques? Celle-ci serait plutôt imputable à leur sous-représentation dans les instances du pouvoir: si les personnalités politiques que l'on voit le plus souvent à la télévision sont des hommes, ce serait tout simplement parce que les hommes politiques sont nettement plus nombreux que les femmes. Ce raisonnement expliquerait la raison pour laquelle, à l'opposé du constat concernant la faible participation des femmes au pouvoir, personne ne semble s'étonner de leur relative absence des plateaux de télévision².

Pourtant, nous savons que la télévision, à l'instar des autres moyens de communication de masse, ne joue pas le rôle de simple miroir mais construit le monde qu'elle nous donne à voir. Avancer cette idée, ce n'est pas souscrire à un constructivisme "idéologique» tel que le conçoit et le critique Gilles Gauthier (2003), selon lequel il y aurait incompatibilité entre le donné et le construit. Le constructivisme dont il est question ici «ne nie aucunement l'existence d'un réel hors du sujet, mais l'intègre au cœur d'une étroite relation où donné et construit s'engendrent mutuellement» (Fleury-Vilatte, Walter, 2004, p. 166). Mais «engendrement mutuel» n'est pas synonyme de simple « fidélité » à un réel préexistant. S'agissant de l'invisibilité des femmes dans les médias, le champ politique n'a rien d'exceptionnel: elles figurent moins souvent que les hommes dans les faits divers (Serdjénian, 1997; Barré, 1999). Faut-il en conclure qu'il leur arrive moins de choses dignes d'intérêt? Tout dépend, bien sûr, de la conception que l'on a de ce qui est « digne d'intérêt», ce qui rejoint les notions d'agenda (McCombs, 2004) ou de cadrage (lyengar, 1999). Nous nous proposons donc d'étudier les mécanismes institutionnels et symboliques qui fonctionnent dans la représentation télévisuelle des femmes politiques et d'évaluer celle-ci à l'aune de leur participation aux instances du pouvoir.

Nous avons choisi, pour ce faire, de nous concentrer sur un genre télévisuel spécifique, le débat, et ce pour plusieurs raisons. Le débat politique étant primordial dans une démocratie, les principales chaines de télévision 3 tirent de telles émissions, qui fait d'elles des arènes démocratiques, une grande partie de leur légitimité. C'est aussi un genre où elles sont relativement autonomes: le débat est organisé sous l'égide des rédactions, qui en contrôlent le contenu et la mise en scène (Neveu, 1995, p. 45). Même si ces émissions suivent l'actualité politique, elles y sont moins directement «collées» que le

2. C'est d'ailleurs la thèse d'Éric Darras dans une étude publiée en 1995 (la même année que notre premier article sur le sujet) : «On ne peut faire grief aux deux journalistes [François-Henri de Virieu et Anne Sinclair] de la remarquable absence des invitations féminines dans leurs émissions. [...] c'est avant tout du fait de leur exclusion objective du gotha politique.» (Darras, 1995 p. 190)

3. Entendons la télévision hertzienne, la seule qui existait pendant une grande partie de la période étudiée et, pour des raisons techniques, économiques et sociologiques, la seule à pouvoir viser un public national. 
journal télévisé. Elles donnent donc à la chaine son image de marque, jouant un rôle dicté par la ligne éditoriale recherchée. Évaluer la participation des femmes politiques à ce genre de débat nous donne une idée de leur accès à la parole politique légitime4.

Nous avons donc procédé à une étude quantitative et diachronique de la participation des femmes politiques aux émissions de débat des principales chaines de télévision françaises. Pour ce faire, nous avons constitué un corpus d'émissions qui, sans être exhaustif, est le plus étendu dans son genre pour ce type d'étude: y figurent 46 émissions différentes, représentant 2441 prestations de diverses personnalités politiques, diffusées entre janvier 1966 (date de la plus ancienne du genre dans les archives de l'INA) et juin 1999 (voir en annexe le corpus des émissions étudiées)5. Par «prestation», nous entendons la participation d'une personnalité à une émission, quelle que soit la durée de celle-ci ou le temps de parole accordé à l'invité. Certes, le dispositif de l'Inathèque à la BNF (site François Mitterrand) permettrait de calculer le temps de parole des différents intervenants et de comparer le temps d'intervention des hommes et celui des femmes plus précisément que nous le faisons ici, à condition toutefois de visionner minutieusement chaque émission. L'avantage de notre approche est qu'elle permet de prendre en compte un très grand nombre d'émissions différentes et de faire une étude statistique diachronique. Car si nous voulons évaluer le raisonnement selon lequel la faible participation des femmes aux instances politiques expliquerait leur faible participation aux émissions de débat télévisé (et réciproquement), il faut comparer différentes périodes pour voir si la progression de la participation dans l'un des domaines correspond ou non à celle de l'autre. Chaque émission représente donc une occasion (prise ou manquée) de participation au débat public et représente une prestation pour chaque participant(e).

\section{La prise de parole légitime dominée par les hommes}

Sur les 2441 prestations qui figurent dans notre corpus, 2141 (87,7 \%) sont masculines et 300 (12,3\%) féminines. Cet écart, qui donne la part du lion aux hommes, reproduit donc la domination masculine du champ politique dans son ensemble. Cependant, comment savoir si ce faible pourcentage d'intervention des femmes à la télévision correspond ou non à leur part dans les instances politiques? Nous rencontrons là des problèmes d'ordre

4. À contraster avec la prise de parole politiquement dévaluée: nous distinguons débat politique et talkshow (voir Neveu, 2005), même s'il y a, au sein du débat, une hiérarchie selon laquelle certaines émissions donnent plus de légitimité à la parole que d'autres, comme nous le verrons plus loin.

5. Il s'agit d'un travail en cours dont la dernière partie, consacrée aux émissions diffusées de 1999 à 2007, reste à compléter mais fait l'objet d’hypothèses à la fin du présent article. 
méthodologique: Quels chiffres représentent la part des femmes dans le champ politique? Ceux de l'Assemblée nationale (dont le record, pour la période qui nous concerne, est de $10 \%$ de femmes), du Sénat (3 à $5 \%$ ), des conseils municipaux (14 à $17 \%$ ), du Parlement européen ( 21 à $30 \%$ ) ou du gouvernement - où la participation des femmes passe de o \%, pour les gouvernements Pompidou entre 1962 et mai 1968, à 31 \% sous Lionel Jospin, entre 1997 et 1999? Ce dernier exemple montre bien les difficultés qui se présentent si l'on considère en bloc toute la période couverte par notre corpus et si nous ne distinguons pas les différents mandats exercés par les femmes.

Pendant cette période, les femmes ont vu leur participation à la vie politique progresser à plusieurs reprises: lors des élections législatives de 1978, dans les gouvernements Mauroy en 1981 et Juppé en 1995, et lors des élections législatives de 1997. Nous avons donc partagé notre corpus en trois périodes qui correspondent plus ou moins à ces moments de progression de la participation des femmes en politique: 1966-1978, 1978-1997 et 1997-1999, ce qui nous donne une progression parallèle de leur participation au débat télévisé: 3,1 \%, $12,7 \%$ et $15,4 \%$ des participants aux émissions de ces trois périodes respectives sont des femmes. Qui plus est, si, en prenant une période donnée, nous comparons le nombre de participantes aux émissions avec le nombre de femmes membres du gouvernement ou députées (un choix qui se justifie par le fait que la plupart de nos intervenantes - $82 \%$ - exercent ou ont exercé ces deux mandats), nous trouvons que la télévision s'en tire relativement bien. De 1983 à 1994, 6,7 \% des ministres et des députés sont des femmes, contre 9,4\% de femmes politiques invitées sur les plateaux de télévision; pour la période 1997-1999, ces chiffres s'élèvent respectivement à 11,3\% et 15,4\%.

Une étude purement statistique laisserait donc supposer que la télévision n'a pas de comptes à rendre et que, malgré un déséquilibre hommes/ femmes accentué, non seulement le pourcentage de la prise de parole féminine à la télévision reflète plus ou moins authentiquement la part des femmes dans les instances politiques et en est donc la conséquence, mais on pourrait dire également, si on y regarde de plus près, que la participation des femmes aux débats télévisés dépasse légèrement leur part dans la vie politique à l'échelon national. Peut-on en conclure que les producteurs d'émissions cherchent à maximiser la participation des femmes, voire qu'ils exercent une sorte de discrimination positive en leur faveur? En fait, ce que nous pourrions appeler des «occasions manquées» viennent infirmer cette hypothèse et incitent à réflexion.

L'accès à ce genre d'émission se fait par la notoriété: il ne suffit pas d'être député, il faut jouer un rôle de premier plan. Ainsi la première participation de Jean-Marie Le Pen à "L'heure de vérité» (13 février 1984) fut-elle controversée, signalant sa consécration, en quelque sorte, et source de légitimité. Il nous semblait donc important de comparer les hommes et les femmes les 
plus en vue, signe de la légitimité acquise par les deux sexes. Pour ce faire, nous avons étudié de plus près les deux émissions jouissant de la plus grande longévité: "Sept sur sept» (TF1, 1981-1997) et "L'heure de vérité» (Antenne 2 / France 2, 1982-1995). Dans les deux cas, Simone Veil est la personnalité la plus fréquemment invitée: elle participe neuf fois à la première émission et cinq fois à la deuxième. Première femme par le nombre de prestations, elle se situe pourtant au treizième rang pour «Sept sur sept», et au quatorzième rang pour "L'heure de vérité». Certes, elle partage ces places avec des hommes politiques de premier plan: Valéry Giscard d'Estaing dans le premier cas, François Bayrou, Jean-Pierre Chevènement et Philippe de Villiers dans le deuxième. Mais en dehors de ces quatre hommes politiques dont la visibilité est égale à celle de la femme politique la plus souvent invitée, pas moins de dix-sept hommes la dépassent en nombre de prestations (et se partagent les vingt-cinq premières places sur les deux chaines). Les femmes sont moins nombreuses et, qui plus est, celles qui investissent le plus les plateaux de télévision le font moins souvent que beaucoup d'hommes.

Il est vrai que cette disparité télévisuelle relève d'une disparité préexistante puisque, comme le note Éric Darras (1995, p. 185), Simone Veil jouit d'un capital politique moins élevé que la plupart des invités de ces deux émissions. Mais ce qui nous intéresse, c'est le fait justement que les producteurs se contentent de reproduire cette inégalité et ne cherchent pas à l'infléchir. Et même s'il est vrai qu'ils jouissent d'une marge de manœuvre restreinte (Darras, 1995, p. 187), il semble qu'ils ne l'exercent pas en faveur des femmes. Sinon, comment expliquer, par exemple, l'absence totale, dans l'ensemble de notre corpus (émissions moins prestigieuses comprises), d'Edwige Avice, pourtant responsable de cinq portefeuilles au sein de sept gouvernements, de 1981 à 1986 et de 1988 à 1992 (huit ans au total), ou de Catherine Lalumière, membre de quatre gouvernements, chargée de trois portefeuilles de 1981 à 1986 ? Le cas le plus révélateur dans ce domaine est celui d'Édith Cresson, la seule à avoir occupé le poste de Premier ministre sans jamais avoir été invitée à "L'heure de vérité», tandis que ses homologues hommes l'ont été à plusieurs reprises: douze fois pour Raymond Barre, neuf pour Jacques Chirac et Édouard Balladur, sept pour Pierre Bérégovoy, Laurent Fabius et Michel Rocard.

D'ailleurs, si l'on compare les différentes émissions, on constate que plus l'émission est considérée comme prestigieuse sur le plan politique ("sérieux», taux d'écoute, heure de diffusion, etc.), moins il y a de femmes politiques qui y participent, et inversement. La plus longue et la plus rigoureusement politique, «L'heure de vérité», ne compte parmi ses invités que 7,1 \% de femmes politiques, bien en-deçà de la moyenne de 12,2 \%, tandis que sa rivale sur la première chaîne, "Sept sur sept», dans laquelle, certaines semaines, les personnalités politiques cèdent la place aux célébrités du showbiz et du cinéma, se rapproche de la moyenne avec $12,4 \%$ de participantes. Une émission qui 
ressemble à «Sept sur sept» de par son dispositif (une revue des informations de la semaine) mais diffusée sur la plus petite chaîne, France 3- «Dimanche soir », de 1994 à 1998, remplacée par « Politique dimanche », de 1998 à 1999-, atteint un taux de 14,4\% de femmes, tandis que «Les quatre vérités» (19951999), toujours sur la deuxième chaîne mais diffusée le matin et de courte durée (10 minutes), se prévaut de $16 \%$ de femmes. Celles-ci sont plus nombreuses aussi dans les émissions de débats que dans celles d'entretiens - on les invite plus fréquemment à une table ronde, parmi plusieurs invités, plutôt qu'individuellement, et on les accepte plus facilement dans le rôle de figurantes que dans celui de personnage principal.

Pourtant, même dans ce moindre rôle, il serait faux de croire que les femmes arrivent à occuper toute la place disponible pour qui chercherait à leur donner un plus grand accès à la parole. Une étude des émissions à thème montre que petit à petit, les femmes ne sont plus exclusivement invitées à parler sur des sujets traditionnellement «féminins » (principalement sociaux: éducation des enfants, santé, jeunesse, etc.), et que dans les années quatreving et quatre-ving-dix, elles commencent à être interrogées sur le commerce ou sur la justice, anciens bastions masculins, tout comme elles arrivent peu à peu à accéder aux ministères correspondants. Malgré cela, l'émission «Polémiques» (1995-1999) montre qu'elles continuent à être associées à des débats sur des sujets traditionnellement féminins ou sur le rôle des femmes dans la société, à raison de 2 contre 1 par rapport aux autres sujets. En fait, 22 des 54 femmes politiques qui participent à cette émission sont là pour débattre de la parité politique, et une émission entière, consacrée au fameux remaniement gouvernemental de novembre 1995, réunit Françoise Giroud et les huit femmes qu'Alain Juppé avait trouvé bon d'écarter. Paradoxalement, donc, sans leur faible participation politique, à cette occasion et sans doute tout au long des années quatre-vingt-dix, la participation des femmes aux émissions politiques aurait été encore plus réduite.

«Polémiques» a joué un rôle significatif dans la campagne pour la parité politique, réunissant femmes de gauche et de droite au-delà de leurs différences idéologiques pour rédiger le Manifeste des dixpour la parité, publié dans L'Express en juin 1996. La participation des femmes à des émissions portant sur un sujet qui les concerne au premier chef marque un net progrès par rapport aux années soixante-dix, quand elles furent exclues d'émissions sur l'avortement («Actuel 2 », le 13 octobre 1972 et le 10 décembre 1973; «Les trois vérités», le 27 mars 1974). Pourtant, dans notre corpus, se trouvent des occasions manquées, même à ce niveau plus modeste - occasions où il est difficile de croire que le faible nombre de femmes occupant un poste approprié suffise à expliquer leur absence: aucune femme sur les 18 invités de "La marche du siècle ", par exemple, qui, le 17 avril 1991, porte sur «La décennie Mitterrand», et seulement deux femmes parmi les 21 invités qui participent à l'émission «Être PS 
en 1990 ». De même pour «Le point sur la table», sur le thème: «Édith Cresson arrive, qu'est-ce qui va changer?», le 16 mai 1991: là encore, aucune femme politique, ni d'ailleurs aucune femme journaliste. Exclure les femmes d'émissions qui portent sur des sujets d'intérêt général, pour moins surprenant que cela puisse paraitre que de les exclure d'émissions qui les concernent spécifiquement, n'en est pas moins dangereux pour leur statut de femmes politiques et pour la légitimité de leur prise de parole politique. Cela sous-entend et laisse accroire que les femmes sont incapables d'exercer un mandat national et non pas sexué, à l'opposé des hommes qui, eux, seraient capables du degré d'abstraction nécessaire pour représenter autrui et non pas leur groupe spécifique.

\section{Logiques du recrutement des « invités » politiques}

L'explication de ces exemples tient aux «logiques du recrutement» (Darras, 1995) dont sont responsables plusieurs acteurs. Malgré le terme «invité», souvent employé pour parler des intervenants, la présence des personnalités politiques dans ces émissions résulte de négociations entre les professionnels des médias, les partis politiques et les conseillers en communication d'individus, voire les individus eux-mêmes. Les personnalités politiques qui jouissent d'un capital politique plus élevé peuvent s'imposer et choisir l'émission ou la chaine qu'elles veulent - renforçant la hiérarchie de celles-ci et, du fait de leur position dominée dans le champ politique, les femmes se trouvent moins souvent que les hommes en mesure d'exercer cette liberté de choix. Il se peut aussi que certaines femmes, réputées pour valoriser l'action et pour critiquer les discours ${ }^{6}$, ne sollicitent pas aussi souvent, ou avec la même insistance, des invitations; mais sans recherches sur le terrain, il est difficile d'évaluer la part de vérité dans ces représentations de soi où le genre est pris à témoin7. Quant aux partis politiques, Janine Mossuz-Lavau les a qualifiés de «cénacles masculins fonctionnant en circuit fermé, se reproduisant à l'identique, et n'étant pas prêts à retirer une place à un homme pour la donner à une femme» (Mossuz-Lavau, 1997, p. 9). Leur rôle dans la désignation de leurs représentants à la télévision doit jouer en faveur des hommes, plus nombreux que les femmes dans les échelons des partis, malgré une certaine ouverture plus récente. Mais il faut dire qu'à quelques exceptions près ${ }^{8}$,

6. Voir, par exemple, Adler, 1993; Saint-Criq, Prévost, 1993; Guigou, 1997.

7. Pour une étude du genre dans la présentation de soi, voir Bonnafous (2002, 2003) et Perry (2005). Nicole Belloubet-Frier (1997), Christine Guionnet (2002) et Magali Della Suda (citée dans Bard et al., 2004) ont étudié le genre et le comportement des femmes politiques, mais sans pour autant établir un lien entre les deux.

8. Dont Paul Amar, transfuge de France 2 sur France 5 et responsable de l'émission «États généraux», trop récente pour figurer dans le corpus dont il est question ici. Exception qui, d'ailleurs, confirme la règle: la chaine éducative est libérée des contraintes d'audience qui pèsent sur France 2 et France 3. 
les équipes rédactionnelles ne se soucient guère du genre dans la constitution de leurs plateaux, pour diverses raisons d'ordre culturel ainsi que structurel et réglementaire.

Les responsables des émissions sont influencés par la course à l'audience, y compris dans le service public. France 2 se voit obligée de justifier la redevance en rassemblant un large public national9. Même si les émissions politiques ciblent un public plus restreint (en général plus âgé et mieux instruit que la moyenne), elles doivent toutefois rassembler le plus grand nombre possible de téléspectateurs. Le sexe des invités n'a sans doute qu'une faible incidence sur la part d'audience d'une émission politique face à un téléfilm ou une émission de divertissement sur une chaine concurrente. Par contre, un invité provocateur attise l'appétit des responsables des rédactions ${ }^{10}$, plus axés sur la controverse que sur les questions de genre, au moins jusqu'aux années quatre-vingtdix quand ces questions deviennent à leur tour sujettes à controverse. Le sexe des invités reste donc un critère de sélection secondaire ${ }^{11}$.

Depuis la création du CSA (Conseil supérieur de l'audiovisuel) en 1982, le rôle des instances de régulation de l'audiovisuel est de "garantir l'égalité entre femmes et hommes». Pourtant, le CSA, ainsi que ses prédécesseurs, fait le relevé des temps de parole des différentes formations politiques ou des différents individus, plutôt que ceux des hommes par rapport à ceux des femmes. En dehors des campagnes électorales, l'accès à la parole et à l'antenne est gouverné par la « règle des trois tiers " $^{12}$; lors des élections législatives ou autres, en fonction des groupes politiques représentés à l'Assemblée nationale; et pendant la campagne présidentielle officielle, les candidats ont droit à un temps d'antenne égal: en d'autres termes, c'est la structure partisane qui règle le droit à l'accès et non pas le sexe des personnalités politiques. Les chaines de télévision sont responsables, devant les instances de régulation, du respect de ces règles et non pas du degré de mixité dans les tables rondes, par exemple.

Les rédactions - majoritairement masculines - restent libres d'évaluer la pertinence de l'égalité des sexes (ainsi que de toutes les variables socioculturelles, en dehors de l'appartenance partisane) dans la constitution des

9. Affirmation confirmée par Hervé Bourges, alors président de France Télévisions, dans l'émission «Informer, éduquer, divertir», BBC 2, 20 octobre 1997.

10. Comme, par exemple, Jean-Marie Le Pen, qui obtient $22,2 \%$ de parts de marché pour «L'heure de vérité » le 27 janvier 1988, contre 13,53\% en moyenne pour cette émission pendant l'année 1987-1988 (chiffres Audimat fournis par France 2). Sur l'ensemble de notre corpus et pendant une période plus courte, Jean-Marie Le Pen participe à 41 émissions, contre 33 pour Simone Veil (la femme qui arrive en tête pour le nombre d'émissions).

11. À notre connaissance, aucune étude n'a été faite sur le rapport entre le sexe des invités et le sexe des téléspectateurs: le public des émissions politiques est majoritairement masculin, à l'instar de leurs participants; y aurait-il un lien de cause à effet?

12. Un tiers du temps d'antenne pour le gouvernement, un tiers pour la majorité parlementaire et un tiers pour l'opposition. Sur une discussion de cette répartition essentiellement quantitative et les propositions du CSA pour une approche plus équitable, voir CSA, 2006. 
plateaux pour les débats. Dans quelle mesure la féminisation de la profession journalistique a-t-elle contribué à une progression de la présence politique féminine dans les émissions de débats télévisés? Elle aurait produit «une grille de lecture plus psychologique des acteurs de la lutte politique» et « un traitement moins déférent, plus distancié, plus ironique parfois » (Neveu, 2000, p. 195, 197), mais a-t-elle augmenté le nombre de femmes politiques interviewées? On doit dire que les émissions présentées par une femme n'incluent pas forcément davantage de femmes politiques que celles présentées par des hommes. "Questions à domicile», par exemple, malgré l'accent mis sur les «intimités publiques» (Le Grignou, Neveu, 1993), attribué au moins en partie à la présence d'une journaliste femme (Anne Sinclair13), ne présente guère plus de femmes que "L'heure de vérité» animée par François-Henri de Virieu $^{14}(7,7 \%$ contre $7,1 \%)$. Si nous comparons trois cas de figure des années quatre-vingt et quatre-vingt-dix ${ }^{15}$, les émissions présentées uniquement par une femme, celles présentées uniquement par un/des homme(s) et celles présentées par les deux sexes, nous constatons qu'avec respectivement $14,6 \%$, $12,7 \%$ et $10,8 \%$ de femmes politiques, les premières dépassent les secondes de deux points pour le pourcentage de participantes. Toutefois, les secondes dépassent les dernières de deux points, et ceci malgré le fait que, parfois, la journaliste se trouve à un niveau hiérarchique supérieur à celui de son coprésentateur masculin. Ces résultats sont pour le moins ambigus quant à l'incidence du sexe des animateurs sur le nombre de femmes politiques ayant accès à ce genre d'émission. Si nous comparons les pourcentages de participation féminine dans chaque émission (plutôt que d'additionner les prestations individuelles ${ }^{16}$ ), nous obtenons exactement la même moyenne pour les émissions ayant une présentatrice que pour celles animées par des hommes (13,7\% dans les deux cas), et seulement un point d'écart entre celles-ci et les émissions présentées par une équipe mixte (12,4\%). Comme nous l'avons montré ci-dessus avec les émissions "L'heure de vérité» (François-Henri de Virieu, 7,1 \% de femmes), «Sept sur sept» (Anne Sinclair, 12,4\%), «Dimanche soir» / "Politique dimanche» (Christine Ockrent et Gérard Leclerc, 14,4\% et $16,7 \%$ ) et «Les quatre vérités» (Gérard Leclerc, 16,2 \%), la variable [chaine + modalités de diffusion (heure, durée, audience espérée)] semble plus pertinente dans ce domaine que celle du sexe des journalistes.

13. D’abord avec Pierre-Luc Séguillon puis, à partir de septembre 1987, avec Jean-Marie Colombani.

14. Avec deux journalistes hommes, Albert du Roy et Alain Duhamel, qui participent régulièrement à l'émission, plus un(e) troisième qui est parfois, mais plutôt rarement, une femme.

15. Puisqu'il y avait très peu de journalistes femmes dans les émissions politiques télévisées des années soixante et soixante-dix, aucune comparaison n'est possible pour cette période.

16. Les deux approches sont valables, mais l'avantage de la deuxième est qu'elle considère l'émission comme un ensemble dont une équipe (féminine, masculine ou mixte) est responsable, tandis que la simple addition des prestations donne la part belle aux titres de longue durée ou ayant plusieurs invités plutôt qu’un(e) seul(e) par émission. 
Si les femmes journalistes sont plus sensibles que les hommes aux questions d'égalité des sexes, il est difficile d'affirmer avec certitude que leur présence explique - plus qu'elle n'accompagne - la progression de la participation des femmes politiques, et ceci pour plusieurs raisons. D'une part, la télévision est le secteur audiovisuel le moins féminisé, avec $25 \%$ de femmes journalistes contre $32 \%$ pour tout l'audiovisuel et $37,5 \%$ pour l'ensemble de la profession (Neveu, 2000, p. 181; 2004, p. 34). Cette situation minoritaire, à laquelle s'ajoutent les logiques de légitimation, n'encourage pas les femmes à déroger aux règles communes dans le secteur. D'autre part, ces règles ont été établies selon les normes universalistes prônant la neutralité par rapport aux origines des représentants de tout genre. Le réflexe de chercher à créer une véritable mixité à toutes les occasions, tel qu'il existe en Grande-Bretagne depuis longtemps, n'existait pas en France dans les années quatre-vingt-dix. Sa légitimité aurait même été contestée. Et effectivement, ce que nous trouvons dans notre corpus des années quatre-vingt-dix, en plein débat paritaire, ce sont des plateaux de femmes pour parler de la parité ou du rôle des femmes dans la société, et des plateaux mixtes, mais à dominante masculine, pour parler d'autre chose.

\section{Égalité, parité, diversité}

Quant aux lois sur la parité, le fonctionnement des rapports entre partis politiques et médias nous incite à croire qu'elles n'auront eu qu'une incidence indirecte et relativement faible sur la participation des femmes au débat télévisé (hypothèse qu'il nous reste à évaluer à l'aune des recherches en cours). Grâce à ces lois, nous avons vu augmenter de façon relativement forte le nombre de conseillères municipales et surtout régionales; au Parlement européen, il y a presque autant de députées que de députés, mais elles étaient déjà relativement nombreuses avant l'introduction de la parité; par contre, le nombre de députées et de sénatrices (comme de femmes maires) reste relativement faible (malgré une lente progression), et il n'y a qu'une présidente de conseil régional. Or, notre corpus nous montre que ce sont ces derniers mandats qui donnent plus souvent accès à la parole politique légitime que les premiers.

Les autres critères de sélection des intervenants - être leader d'un parti ou d'un mouvement, être candidat à la présidence ou membre d'un gouvernement - ne sont pas régis par les lois sur la parité. Si, depuis 2000, le pourcentage de femmes au sein des gouvernements successifs atteint parfois les 33 \%, c'est grâce au débat sur la parité, puisque la loi n'en dit rien; le fait que ce chiffre a baissé durant les mandats de Jean-Pierre Raffarin et de Dominique de Villepin de 2004 à 2007 montre qu'aucune progression n'est garantie. Le nombre de femmes leaders n'a pas augmenté non plus: en 2008, Martine 
Aubry est arrivée à la tête du Parti socialiste, certes, mais Michèle Alliot-Marie, la première femme à diriger un grand parti, a été remplacée en 2002, quand le RPR a cédé la place à l'UMP. Quant aux candidates à l'élection présidentielle, elles n'étaient pas plus nombreuses en 2007 qu'en 2002, mais, du fait du nombre inférieur de candidats masculins, leur pourcentage était plus élevé: $30 \%$ au lieu de $25 \%$. À cette augmentation relative s'ajoute un élément qualitatif: Ségolène Royal représentait un grand parti et elle était candidate au deuxième tour, deux facteurs qui auront joué en faveur de sa présence à l'antenne. Pourtant, dans l'ensemble, il s'agit d'une progression plutôt lente et fluctuante. Nous nous croyons donc en mesure de postuler que, pour la période de 1999 à nos jours, l'on constatera une progression quantitative des femmes semblable à celles repérées dans le présent corpus, et non pas une hausse soudaine, grâce à la parité.

Depuis lors, la notion d'indifférence à l'indifférence, longuement débattue dans les années quatre-vingt-dix, a cédé la place non seulement à la parité mais aussi à la "[promotion de] la diversité», qui s'applique entre autres à l'audiovisuel. Le CSA s'est vu doter, en 2006, d'une nouvelle mission afin de veiller au respect de ce nouveau principe. Désormais, les responsables de la télévision doivent faire en sorte que l'accès à l'antenne soit équitablement réparti entre les différents groupes socioculturels. Le problème, en ce qui concerne l'égalité des sexes, c'est que le genre va devoir trouver sa place parmi d'autres variables identitaires, désignées par le mot fourre-tout «diversité». Même si la nomination au gouvernement de femmes d'origines diverses, telles Rachida Dati, Rama Yade et Fadela Amara, et l'accès à l'antenne qui s'ensuit, ont permis jusqu'ici de concilier les deux missions, on peut se demander si l'égalité des sexes ne risque pas de se trouver reléguée au deuxième plan, car considérée comme acquise face à de nouveaux défis comme l'origine ethnique, les «minorités visibles » attirant l'attention des créateurs d'images que sont les professionnels de la télévision.

Il ressort de notre analyse qu'entre 1966 et 1999, la télévision donne à voir de plus en plus de femmes politiques, épousant les évolutions qui se produisent au sein de la classe politique, mais qu'il en résulte une sous-représentation des femmes qui renforce et légitime celle qui existe dans le champ politique. Ceci se traduit par une participation minoritaire des femmes au débat politique, sauf pour certains domaines considérés comme étant de leur ressort en tant que femmes. Les femmes politiques se trouvent donc, à l'instar des femmes en général, définies et marquées par leur appartenance au sexe féminin. Malgré une légère amélioration de leur participation, tant sur le plan du nombre que dans le domaine des sujets qu'elles débattent, le fonctionnement du champ «médias audiovisuels / partis politiques» nous laisse anticiper les résultats du travail qu’il reste encore à faire sur les années 1999-2009, 
et nous avançons l'hypothèse selon laquelle nous constaterons un prolongement de cette lente progression, plutôt qu'un changement brutal et marqué, pour les années qui ont suivi l'adoption de la parité. Celle-ci, déjà limitée dans ses effets au sein du champ politique, se fera sans doute encore moins sentir sur les plateaux de télévision, sauf dans la mesure où la parité elle-même reste un sujet de débat.

Notre analyse nous amène à constater que dans le domaine de l'égalité entre les sexes, la télévision suit le mouvement et ne le mène pas. Sa mission informative et éducatrice, au nom de laquelle elle cherche à former l'opinion, se limite à offrir un espace public où le débat sur cette égalité peut avoir lieu - ce qui n'est pas sans importance -, mais l'institution audiovisuelle ellemême ne semble pas se constituer en modèle de bonne pratique en la matière, et le système institutionnel et réglementaire ne l'incite guère à le devenir. Étant donné son rôle de «caisse de résonance» (Coulomb-Gully, 1994, p. 237), elle devient donc de facto un vecteur d'entretien de stéréotypes, reproduisant un monde politique fait par et pour les hommes qui, grâce à cette reproduction, semble aller de soi (sauf lors d'émissions spécialement conçues pour, justement, débattre de la question). Épousant les évolutions sociales, elle devient petit à petit plus ouverte aux femmes, mais reste néanmoins touchée par le "sexisme ordinaire », celui qui est inconscient, non voulu. La télévision a, tout simplement, d'autres priorités.

Cet état de choses va-t-il perdurer? La promotion de la diversité est venue se greffer aux exigences d'audience et d'équilibre partisan qui, avec les vicissitudes de l'actualité, régissaient jusqu'ici les logiques du recrutement du personnel politique dans les émissions de débat. Dans la mesure où elle rend légitime la prise en compte de l'identité socioculturelle des invités comme critère de sélection, cette nouvelle mission pourrait favoriser la constitution de plateaux mixtes, surtout au fur et à mesure que le personnel des rédactions se féminise. Pourtant, après un débat sur le périmètre de la diversité, le CSA $\mathrm{a}$ « tout d'abord considéré que son action devait porter de manière prioritaire, mais non exclusive, sur la diversité des origines» (CSA, 2008, p. 19). Une fois de plus, la promotion des femmes est occultée en faveur d'autres objectifs ${ }^{17}$. Reste à savoir si cette priorité accordée à l'origine ethnique se fera aux dépens de l'égalité des sexes, ou si elle accompagnera celle-ci, dans le domaine de l'accès à la parole politique légitime.

17. En fait, la loi sur l'égalité des chances (2006) ne fait qu'entériner un mouvement vers une meilleure représentation des «minorités visibles» à la télévision, entrepris à l'initiative du CSA en 2001, répondant aux accusations d'associations telles que le Collectif Égalité ou le Club Averroès (Voir FASILD, 2004). Cette action volontariste en faveur des minorités ethniques contraste avec l'inaction du CSA dans le domaine de l'égalité des sexes et donne à l'entreprise de meilleures chances d'aboutir. 


\section{Références}

AD LER Laure, 1993, Les femmes politiques, Paris, Le Seuil.

BARD Christine et al., 2004, Quand les femmes s'en mêlent. Genre et pouvoir, Paris, La Martinière.

BARRÉ Virginie et al., 1999, Dites-le avec les femmes. Le sexisme ordinaire dans les médias, Paris, CFD/AFJ (Centre de formation et de documentation / Association des femmes journalistes).

Belloubet-Frier Nicole, 1997, «Sont-elles différentes ? », Pouvoirs, n 82, p. 59-75.

BonNAFOUS Simone, 2002, "La question du genre et de l'ethos en communication politique », Actes du premier colloque franco-mexicain des sciences de la communication, Mexico, 8-10 avril 2002, [en ligne] [URL: http://edutice.archives-ouvertes.fr/ docs/00/00/18/55/PDF/actes.pdf], site consulté le 26 février 2009.

- 2003, "Femme politique” : une question de genre?» Réseaux, n 120, p. 121-145.

Coulomb-Gully Marlène, 1994, Radioscopie d'une campagne, Paris, Kimé.

CSA, 2006, «Réflexions sur les modalités du pluralisme», Assemblée plénière du 18 juillet 2006, dossier d'actualité mis en ligne le 2 août 2006 [URL: http://www.csa. fr/actualite/dossiers/dossiers_detail.php?id=118335], site consulté le 26 février 2009.

- 2008, «Le Conseil évalue la représentation de la diversité dans les médias audiovisuels ", La Lettre du CSA, n²16, p. 15-19.

DARRAS Éric, 1995, "Le pouvoir “médiacratique” ? Les logiques du recrutement des invités politiques à la télévision », Politix, n³0, p. 183-198.

Derville Grégory, Pionchon Sylvie, 2005, "La femme invisible. Sur l'imaginaire du pouvoir politique», Mots. Les langages du politique, $n^{\circ} 78$, Usages politiques $d u$ genre, p. 53-64.

FASILD (Fonds d'aide et de soutien pour l'intégration et la lutte contre les discriminations), 2004, Écrans pâles. Diversité culturelle et culture commune dans l'audiovisuel. Actes du colloque du 26 avril 2004, Paris, La Documentation française.

FLeuRY-VILATTE Béatrice, WAlter Jacques, 2004, "Des usages du constructivisme», Questions de communication, $\mathrm{n}^{\circ}$ 5, p. 161-168.

Gauthier Gilles, 2003, "Critique du constructivisme en communication », Questions de communication, $\mathrm{n}^{\circ}$ 3, p. 185-198.

GuIGou Élisabeth, 1997, Être femme en politique, Paris, Plon.

GUIONNET Christine, 2002, «Entrées de femmes en politique. L'irréductibilité du genre à l'heure de la parité», Politix, $n^{\circ}$ 60, p. 113-146.

Holmes Diana, 1988, "The Madonna and the dragon: the representation of women politicians in the french press», Modern and Contemporary France, $\mathrm{n}^{\circ} 33$, p. 10-16.

IYENGAR Shanto, 1999, Is Anyone Responsible? How Television Frames Political Issues, Chicago, Chicago University Press.

LE GRIG Nou Brigitte, NEVEU Éric, 1993, «Intimités publiques. Les dynamiques de la politique à la télévision », Revue française de science politique, $n^{\circ}$ 43, p. 940-969. 
McCombs Maxwell, 2004, Setting the Agenda. The Mass Media and Public Opinion, Cambridge, Polity Press.

Mossuz-LAvau Janine, 1997, "Les Françaises et la politique. De la citoyenneté à la parité », Regards sur l'actualité, $\mathrm{n}^{\circ}$ 236, décembre, p. 3-14.

NEVEU Éric, 1995, «Les émissions politiques à la télévision. Les années quatre-vingt ou les impasses du spectacle politique», Hermès, n 17-18, p. 145-162.

- 2000, «Le genre du journalisme. Des ambivalences de la féminisation d’une profession », Politix, n ${ }^{\circ}$ 1, p. 179-212.

- 2004, Sociologie du journalisme, Paris, La Découverte.

- 2005, "Politicians without politics. A polity without citizens: the politics of the chat show in contemporary France », Modern and Contemporary France, $n^{\circ} 13$ (3), p. 323-335.

Perry Sheila, 1995, "French television and women politicians», Women and Representation, J. Still, D. Knight éd., Nottingham, Nottingham University Press, p. 21-42.

- 2000, "Women politicians and television. The numbers game», Web Journal of French Media Studies, $n^{\circ} 3$ (1), octobre, [en ligne] [URL: http://wjfms.ncl.ac.uk/ perrWJ.htm], site consulté le 26 février 2009.

- 2005, "Gender difference in french political communication: from handicap to asset? ", Modern and Contemporary France, $\mathrm{n}^{\circ} 13$ (3), p. 337-352.

SAINT-CRIQ Régine, PrÉvost Nathalie, 1993, Vol au-dessus d'un nid de machos, Paris, Albin Michel.

SERdJÉnIAn Évelyne éd., 1997, Femmes et médias. Actes du xve Congrès de l'Union professionnelle féminine, Toulon, 4-8 octobre 1995, Paris, L'Harmattan.

SouRd Cécile, 2005, "Femmes ou politiques? La représentation des candidats aux élections françaises de 2002 dans la presse hebdomadaire», Mots. Les langages du politique, $\mathrm{n}^{\circ} 78$, Usages politiques du genre, p. 65-77.

\section{Annexe}

\section{Corpus d'émissions étudiées}

\begin{tabular}{l|c|c|c|c|c}
$\begin{array}{l}\text { Émissions } \\
\text { Dates de diffusion }\end{array}$ & Chaine & $\begin{array}{c}\text { Invité(es) } \\
\text { politiques }^{18}\end{array}$ & $\begin{array}{c}\text { Politiques } \\
\text { hommes }\end{array}$ & $\begin{array}{c}\text { Politiques } \\
\text { femmes }\end{array}$ & $\begin{array}{c}\text { Femmes } \\
\%\end{array}$ \\
\hline $\begin{array}{l}\text { Face à face } \\
\text { janvier 1966 / septembre 1966 }\end{array}$ & 1 & 7 & 7 & 0 & 0 \\
$\begin{array}{l}\text { En direct avec } \\
\text { octobre 1966 / avril 1968 }\end{array}$ & 1 & 9 & 9 & 0 & 0 \\
$\begin{array}{l}\text { Les dossiers de l'écran } \\
\text { avril 1967 / septembre 1991 }\end{array}$ & 2 & 28 & 27 & 1 & 3,6 \\
$\begin{array}{l}\text { Face à face } \\
1969 \text { / décembre 1969 }\end{array}$ & 1 & 10 & 10 & 0 & 0
\end{tabular}

18. Seuls les invités politiques français sont pris en compte. 
Les fermmes politiques à la télévision

\begin{tabular}{|c|c|c|c|c|c|}
\hline $\begin{array}{l}\text { Émissions } \\
\text { Dates de diffusion }\end{array}$ & Chaine & $\begin{array}{l}\text { Invité(es) } \\
\text { politiques }\end{array}$ & $\begin{array}{l}\text { Politiques } \\
\text { hommes }\end{array}$ & $\begin{array}{l}\text { Politiques } \\
\text { femmes }\end{array}$ & $\begin{array}{c}\text { Femmes } \\
\%\end{array}$ \\
\hline $\begin{array}{l}\text { À armes égales } \\
\text { février } 1970 \text { / mars } 1973\end{array}$ & 1 & 49 & 49 & 0 & 0 \\
\hline $\begin{array}{l}\text { Hexagone } \\
\text { mars } 1970 \text { / septembre } 1972\end{array}$ & 1 & 10 & 9 & 1 & 10,0 \\
\hline $\begin{array}{l}\text { L'actualité en question } \\
\text { janvier } 1971 \text { / juillet } 1972\end{array}$ & 1 & 12 & 12 & 0 & 0 \\
\hline $\begin{array}{l}\text { L'heure de vérité } \\
\text { janvier } 1972 \text { / décembre } 1972\end{array}$ & $1 / 2$ & 6 & 6 & 0 & 0 \\
\hline $\begin{array}{l}\text { Actuel } 2 \\
\text { septembre } 1972 \text { / novembre } \\
1974\end{array}$ & 2 & 52 & 51 & 1 & 1,9 \\
\hline $\begin{array}{l}\text { Les trois vérités } \\
\text { mai } 1973 \text { / juillet } 1974\end{array}$ & 1 & 22 & 20 & 2 & 9,1 \\
\hline $\begin{array}{l}\text { L'avenir du futur } \\
\text { mars } 1975 / 1987\end{array}$ & 1 & 7 & 6 & 1 & 14,3 \\
\hline $\begin{array}{l}\text { L'huile sur le feu } \\
\text { septembre } 1976 \text { / juillet } 1977\end{array}$ & 2 & 17 & 14 & 3 & 17,6 \\
\hline $\begin{array}{l}\text { L'homme en question } \\
\text { octobre } 1976 \text { / septembre } 1978\end{array}$ & 3 & 9 & 9 & 0 & 0 \\
\hline $\begin{array}{l}\text { Cartes sur table } \\
\text { avril } 1977 \text { / mars } 1981\end{array}$ & 2 & 34 & 31 & 3 & 8,8 \\
\hline $\begin{array}{l}\text { Le grand débat } \\
\text { septembre } 1980 \text { / novembre } \\
1981\end{array}$ & 1 & 27 & 24 & 3 & 11,1 \\
\hline $\begin{array}{l}\text { Sept sur sept } \\
\text { septembre } 1981 \text { / juillet } 1997\end{array}$ & 1 & 355 & 311 & 44 & 12,4 \\
\hline $\begin{array}{l}\text { Droit de réponse } \\
\text { décembre } 1981 \text { / septembre } \\
1987\end{array}$ & 1 & 48 & 39 & 9 & 18,8 \\
\hline $\begin{array}{l}\text { L'heure de vérité } \\
\text { mai } 1982 \text { / juin } 1995\end{array}$ & 2 & 238 & 221 & 17 & 7,1 \\
\hline $\begin{array}{l}\text { Politiques } \\
\text { février } 1984 \text { / mai } 1984\end{array}$ & 1 & 9 & 8 & 1 & 11,1 \\
\hline $\begin{array}{l}\text { Face à la Trois } \\
\text { janvier } 1985 \text { / avril } 1986\end{array}$ & 3 & 27 & 24 & 3 & 11,1 \\
\hline $\begin{array}{l}\text { Questions à domicile } \\
\text { mars } 1985 \text { / mai } 1989\end{array}$ & 1 & 52 & 48 & 4 & 7,7 \\
\hline $\begin{array}{l}\text { Le monde en face } \\
\text { septembre } 1987 \text { / mars } 1988\end{array}$ & 1 & 12 & 10 & 2 & 16,7 \\
\hline $\begin{array}{l}\text { Médiations } \\
\text { septembre } 1987 \text { / juin } 1992\end{array}$ & 1 & 39 & 30 & 9 & 23,1 \\
\hline $\begin{array}{l}\text { La marche du siècle } \\
\text { septembre } 1987 \text { à } 1999\end{array}$ & $2 / 3$ & 116 & 105 & 11 & 9,5 \\
\hline $\begin{array}{l}\text { Forum FR 3-RMC } \\
\text { janvier } 1988 \text { / février } 1990\end{array}$ & 3 & 18 & 16 & 2 & 11,1 \\
\hline $\begin{array}{l}\text { Aparté } \\
\text { octobre } 1989 \text { / décembre } 1990\end{array}$ & 5 & 10 & 9 & 1 & 10,0 \\
\hline
\end{tabular}




\begin{tabular}{|c|c|c|c|c|c|}
\hline $\begin{array}{l}\text { Émissions } \\
\text { Dates de diffusion }\end{array}$ & Chaine & $\begin{array}{l}\text { Invité(es) } \\
\text { politiques }\end{array}$ & $\begin{array}{l}\text { Politiques } \\
\text { hommes }\end{array}$ & $\begin{array}{l}\text { Politiques } \\
\text { femmes }\end{array}$ & $\begin{array}{c}\text { Femmes } \\
\%\end{array}$ \\
\hline $\begin{array}{l}\text { Le point sur la table } \\
\text { octobre } 1990 \text { / janvier } 1993\end{array}$ & 1 & 47 & 46 & 1 & 2,1 \\
\hline $\begin{array}{l}\text { Direct } \\
\text { septembre } 1991 \text { / juin } 1992\end{array}$ & 2 & 21 & 16 & 5 & 23,8 \\
\hline $\begin{array}{l}\text { Durand la nuit } \\
\text { septembre } 1992 \text { / juin } 1993\end{array}$ & 1 & 41 & 38 & 3 & 7,3 \\
\hline $\begin{array}{l}\text { Repères } \\
\text { octobre } 1992 \text { / décembre } 1993\end{array}$ & 3 & 15 & 9 & 6 & 40,0 \\
\hline $\begin{array}{l}\text { Zone interdite } \\
\text { mars } 1993 / \text { juin } 1995\end{array}$ & 6 & 11 & 10 & 1 & 9,1 \\
\hline $\begin{array}{l}\text { Je suis venu vous dire... } \\
\text { octobre } 1994 \text { / juin } 1995\end{array}$ & 1 & 31 & 27 & 4 & 12,9 \\
\hline $\begin{array}{l}\text { Dimanche soir } \\
\text { octobre } 1994 \text { / juin } 1998\end{array}$ & 3 & 111 & 95 & 16 & 14,4 \\
\hline $\begin{array}{l}\text { Les quatre vérités } \\
\text { janvier } 1995^{19} / \text { mai } 1997\end{array}$ & 2 & 266 & 223 & 43 & 16,2 \\
\hline $\begin{array}{l}\text { Face à la une } \\
\text { janvier } 1995 \text { / avril } 1995\end{array}$ & 1 & 15 & 14 & 1 & 6,7 \\
\hline $\begin{array}{l}\text { La revue de campagne } \\
\text { de Michèle Cotta } \\
\text { janvier } 1995 \text { / mars } 1995\end{array}$ & 2 & 36 & 33 & 3 & 8,3 \\
\hline $\begin{array}{l}\text { Carnet de campagne } \\
\text { janvier } 1995 / \text { mai } 1995\end{array}$ & 2 & 11 & 9 & 2 & 18,2 \\
\hline $\begin{array}{l}\text { La France en direct } \\
\text { janvier } 1995 \text { / avril } 1995\end{array}$ & 2 & 12 & 11 & 1 & 8,3 \\
\hline $\begin{array}{l}\text { Invité spécial } \\
\text { septembre } 1995 \text { / juin } 1996\end{array}$ & 2 & 29 & 27 & 2 & 6,9 \\
\hline $\begin{array}{l}\text { Polémiques } \\
\text { septembre } 1995 \text { / avril } 1999\end{array}$ & 2 & 328 & 266 & 62 & 18,9 \\
\hline $\begin{array}{l}\text { Franchement } \\
\text { septembre } 1996 \text { / avril } 1997\end{array}$ & 2 & 16 & 14 & 2 & 12,5 \\
\hline $\begin{array}{l}\text { France Europe Express } \\
\text { octobre } 1997 \text { / juin } 1999^{20}\end{array}$ & 3 & 16 & 14 & 2 & 12,5 \\
\hline $\begin{array}{l}\text { Public } \\
\text { septembre } 1997 \text { / juin } 1999\end{array}$ & 1 & 105 & 89 & 16 & 15,2 \\
\hline $\begin{array}{l}\text { Mots croisés } \\
\text { septembre } 1997 \text { / juin } 1999\end{array}$ & 2 & 64 & 59 & 5 & 7,8 \\
\hline $\begin{array}{l}\text { Politique dimanche } \\
\text { septembre } 1998 \text { / juin } 1999\end{array}$ & 3 & 36 & 30 & 6 & 16,7 \\
\hline $\begin{array}{l}\text { Direct } \\
\text { février } 1999 \text { / mai } 1999\end{array}$ & 2 & 7 & 6 & 1 & 14,3 \\
\hline Ensemble des émissions & & 2141 & 2141 & 300 & 12,3 \\
\hline
\end{tabular}

19. Date à partir de laquelle nous disposons des informations nécessaires.

20. Date limite du corpus. 